\title{
EDITORIAL
}

\section{A Radical Approach to Medical Education - A British Perspective}

This review is informed by current postgraduate training of doctors in the UK, emphasising changes as to how this training is being delivered, and the objectives in ensuring that doctors are prepared to meet the challenges of patient and community expectations, and are responsive to the requirements of a modern society and concerns regarding the sometimes conflicting demands of autonomy, the wish to do good but not harm, respect for beliefs, equity and equality. There is also a need to ensure that medical talent is not wasted and that the skills and knowledge of trainees are developed and utilised efficiently..

There is therefore a requirement that educational training opportunities should be maximised, and that training should have primacy over service delivery for trainees, and that there should be a clear curriculum for trainees with clear achievable outcomes. Trainees should be imbued with a culture of lifelong learning, especially with the rapid development of medical knowledge and clinical care. Indeed, this vast expansion of medical knowledge makes it impossible for trainees to memorise and recall as perhaps previously possible, and although a knowledge of certain basic principles and the ability to deal with common practical issues remain, depending on the stage and speciality of the trainee, of equal importance is to develop the skills to locate and evaluate knowledge, and apply appropriately to the clinical situation ${ }^{1}$.

The traditional scientific background to medical training remains essential, particularly for those who are likely to pursue a career in academic medical science. However, to improve population health, a broader skill development is required to integrate with other health professionals, appreciate the perspective of patients, and how their social situation inter-relates to their illness, and understand the ethical context in which doctors work. Indeed, a broader understanding of health, rather than just illness is required.
This has consequences for medical personnel of all grades and other health care personnel. In particular, senior staff have a professional commitment to training their junior staff (as expected by all the Speciality Colleges), and accept that more clinical care will need to be provided by seniors. To achieve this, the pyramidal structure of medical careers, needs to be inverted, with an expansion of senior doctors, and maximisation of the utilisation of the clinical skills of other health care workers. This will ensure that patients are cared for by staff appropriately trained and skilled, for the situation that they are in. Streamlining medical training ensures that trainees are efficiently developed to their potential with minimum wastage of talent.

A curriculum based on continuous formative competency-based assessment, to enable trainees to address deficiencies of specific competencies, as they progress, does not necessarily remove the requirement for summative assessment, or examinations, as a benchmark of standards prior to entering independent practice, or more specialised training, but should include assessment of practical skills, such as interaction with patients, and efficient use of resources. For example, a viva question might relate to a diagnostic clinical scenario, where the answer should not be a text-book list, but the probable diagnoses in that particular patient, given their age, sex, environment and other features specific to that patient. Management should also be discussed within that context.

To avoid wastage of medical trainees as a resource, failure to progress to the next stage should be the exception rather than the rule. Well-structured formative training, with appropriate career advice will achieve this. This requires that trainees are supervised clinically, and also in respect of their personal development.

Training needs to be closely integrated with service provision, stand alone courses have a value, but should be mainly concerned with aspects of the curriculum, or occasionally with particular interests of the trainee, 
whilst viva skills, and other life skills such as interview techniques, should be a small part of the educational process. The prime objective should be to train doctors skilled in the arts and science of medicine, able to play a role in modern medical practice, to interact constructively with patients, and be able to relate to other health professionals, and appreciate their responsibility to society rather than produce doctors who can pass examinations, but have not developed other essential personal and professional skills.

Advantages of a competency-based formative assessment education:

\section{For trainees:}

A clear career pathway, of defined duration, meeting the achievable aspirations of the trainee.

Confidence in possessing fundamental practical and other skills.

Satisfaction as a team member.

Flexibility to respond to the rapidly developing scientific aspects of medicine, new diseases, and previously unfamiliar social and public health situations.

More time-efficient training, less time spent on noneducationally-useful activities.

\section{For trainers:}

The opportunity to bring out the best in trainees, and ensure a legacy of a well- trained medical workforce.

Share and experience new approaches to patient care with trainees.

\section{For patients:}

The reassurance of being cared for by a well-trained medical team of sufficient seniority.

Medical and other carers within the team, who are responsive to all aspects of the patient's concerns.

\section{For Public Health:}

Trainees responsive to the social, environmental and Public Health aspects of health and disease.

\section{For Government:}

A cost-effective training programme, minimising waste, producing doctors fit forpurpose, who are flexible and responsive to the Health needs of society, and work constructively with colleagues and other health professionals.

\section{Disadvantages (possible or perceived):}

Trainees:

Frustration for those who wish to proceed quickly to more specialist training ${ }^{2}$.

\section{Trainers and senior doctors:}

More time required for training (formal and opportunistic).

Perception that trainees are inadequately trained (at least within their speciality - speciality skills develop later), and require more support.

More hands on care, including emergency care, required from Consultants.

\section{Patients:}

Less continuity of care from junior doctors, due to shorter rotations, and educational requirements. The European working time directive, which is not unrelated to providing good education, is a major factor in the UK, affecting continuity of care.

\section{Government:}

More medical or other health care staff required to provide routine care.

More equitable care provision will identify funding gaps.

\section{Postgraduate Medical Training in the United Kingdom}

In 2000, following a commitment to modernise the Senior House Officer (SHO) grade, a report "Unfinished Business" was presented by Liam Donaldson, Chief Medical Officer, outlining the unsatisfactory situation of the SHOs, in respect of training and career prospects ${ }^{3}$. Many trainees (including a disproportionate number of non-UK graduates) were stuck at this grade, with little supervision or training and poor prospects for furthering their career. As a consequence, a new system of recruitment and training, Modernising Medical Careers (MMC), was introduced, commencing in 2005.

Trainees, preference being given to European Union medical students (a requirement of UK membership of the European Union), but mainly new UK graduates, were appointed to two-year Foundation Training Programmes, through a standardised recruitment and assessment system ${ }^{4}$. Rotations consisted of four months in different specialities, to include at least General Medicine. 
Achievement of the national curriculum competences had to be demonstrated each year to progress to the next year. There was also a formal teaching programme, and opportunistic teaching was encouraged. The aim was for trainees to develop generic skills and competencies, and knowledge from undergraduate years in the first year, the second year was to demonstrate an ability to assess and manage the acutely ill patient, and to improve generic skills of teamwork, time management, communication and self-directed learning. Particularly in the second year, there was an opportunity to be placed in health care settings other than hospitals, such as public health, primary care and academia.

Therefore, despite rotating through different specialities, the standard curriculum competences had to be achieved.

In addition, the Programme was to provide trainees with the opportunity to sample specialities that they might favour as a career, and to broaden the outlook of those determined to follow a specific career pathway before they entered speciality training. These aims could be in conflict, and in reality, there were practical issues since placements were based on existing posts, there was some funding for additional innovative placements and primary care, but insufficient to achieve everything hoped for in the initial plans.

The second year in particular, has come under considerable criticism from many trainees and senior staff, because of the lack of specialist skills they possess for a particular placement. Some rotations lack flexibility and some trainees are impatient to experience their intended speciality, particularly as the selection process for specialities begins early in the second year. These trainees are now allowed and sometimes encouraged to take initial specialist College examinations early in their first year. It is likely that the conflict between the Colleges and many trainees, and the Educational principles behind the Foundation Programme, will reach a compromise, by the second year becoming "themed", that is consisting largely of a particular speciality. This is in conflict with the objectives of producing doctors with a broad-based understanding of and commitment to health care within society.

Specialist training also has a curriculum for each speciality, in many cases there is the opportunity for even more specialised experience towards the end. Most Speciality Programmes now run for seven or eight years (longer than originally hoped for by Government). Some specialities have only one appointment process at the beginning, others such as medicine, have two or three years core speciality training, followed by a further competitive application for more specialist training, such as Cardiology 5 .

There is opportunity for non-European Union doctors to enter specialist training within the UK, but this not easy, and discussion is not within the scope of this Editorial.

Trainees with particular issues, such as a disability, have, at least theoretically, the option of more flexibility within their programmes. Flexible training should add more equity and diversity to the mix of trainees. Overall, doctors should mirror in other ways the make-up of society, such as similar proportions in sex ratio, ethnicity and social class. Equal sex ratio has been achieved, if not exceeded in the UK, some, but not all, ethnic groups are proportionally represented, but with social class origin, the disadvantaged are poorly represented ${ }^{6}$. These issues can be ameliorated by flexible training, although the solution lies with Medical School selection and preUniversity education.

\section{Summary}

The advantages and problems of competency-based medical training have been discussed, as developed in the UK. Although only postgraduate and pre-specialist training has been described, training is regarded as a continuum, from entering medical school to completion of medical practise, and the issues of continuous professional development and revalidation relate to this. The UK model will continue to develop, and whilst responding positively to constructive criticism from within the profession, hopefully, will not be deterred from ensuring that doctors are suitably trained to meet the expectations of civilised society, unfortunately, the experiences of the regressive changes to the second Foundation year highlights that impetus for radical change could be lost. Implementation, especially within a resource-poor environment, may well be costly, and change will be threatening to more established clinicians, but streamlined training, producing doctors better able to face the challenges ahead, and avoid the wastage of trainees who fail to complete, or have very prolonged unsatisfactory training, has great advantages to trainees and society, compared to a traditional highly competitive pyramidal career structure. It has been suggested that excellence is being sacrificed to deliver competence, but a well-structured programme can produce both, including high academic achievement. 
Fenk, Chen, Bhutta et $\mathrm{al}^{7}$, in a report commissioned by The Lancet, proposed a global approach to reform medical education. Commenting on the failure of medical care to address the needs of most populations, they supported competency-based curricula, responsive to changing needs and local contexts, and promotion of inter-professional education, especially for training in generic skills. Information technology can now support learning across national boundaries, and professions, which could lead to internationally recognised common accreditation. Increasingly, in some countries, medical training is moving towards this goal, being patientcentred but with a commitment to the needs of society, in others there is still a way to go ${ }^{8}$. To achieve a global health care system, responsive to need, will require considerable maturity from politicians, the professions and the public. Enthusiasm by medical students may well help to push this agenda forward ${ }^{9}$.

(J Bangladesh Coll Phys Surg 2011; 29: 58-61)

\section{R H Robson}

Address for correspondence : R H Robson FRCPE, Retired Consultant Physician and Cardiologist, Cumbria, UK. howard.robson@doctors.org.uk

\section{References}

1. UK General Medical Council. Tomorrow’s doctors: outcomes and standards forundergraduate medical education.London:General Medical Council. 2009.

2. Bowness JS, Clift B. Foundation year doctors lack surgical experience. Br Med J 2011; 342:173.

3. Unfinished Business. Proposals for Reform of the Senior House Officer Grade. 2002. Available at : www.scotland.gov.uk/Resource/Doc/46951/0013974.pdf Accessed January 16, 2011.

4. The Foundation Programme. Available at: www.foundationprogramme.nhs.uk/pages/home Accessed January 16, 2011.

5. Medical Speciality Training (England). Available at: www.mmc.nhs.uk/ Accessed January 162011.

6. Equality and Diversity. Available at:www.dh.gov.uk/en/ Managingyourorganisation/Workforce/Equalityanddiversity/ index.htm Accessed January16 2011.

7. Frenk J, Chen L, Bhutta ZA et al. Health professionals for a new century: transforming education to strengthen health systems in an interdependent world. Lancet 2010; 376: 192358.

8. Horton R. A new epoch for health professionals' education. Lancet 2010; 376: 1875-7.

9. Stigler FL, Duvivier RJ, Weggemans M, Salzer HJF. Health professionals for the $21^{\text {st }}$ century: a students' view. Lancet 2010; 376:1877-8. 